\title{
Developing thymocytes roll the Dice(r)
}

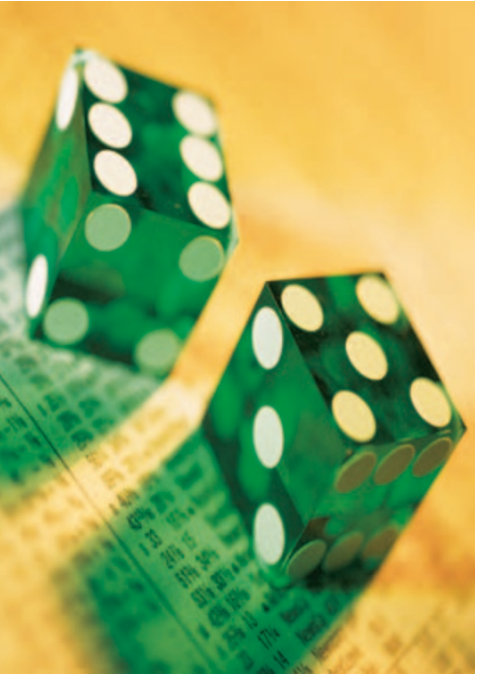

Micro-RNAs (miRNAs) and small interfering RNAs (siRNAs) - which are short (21-22-nucleotide) RNAs that are generated from longer precursors by the ribonuclease III enzyme Dicer - regulate gene transcription in many organisms and have been proposed to have a role in controlling haematopoiesis. Now, a report published in The Journal of Experimental Medicine shows that Dicer is required for the generation and survival of $\alpha \beta$-T-cell receptor $(\alpha \beta-\mathrm{TCR})^{+} \mathrm{T}$ cells.

It is estimated that one in three mRNAs are regulated by miRNAs, and several pieces of evidence indicate that miRNAs regulate haematopoiesis.
Mice that are constitutively deficient in Dicer die in utero, so to investigate the role of Dicer-generated small RNAs in T-cell development, Cobb et al. generated mice in which Dicer was specifically eliminated at the early stages of T-cell development. Mice in which Dicer was eliminated at the double negative 3 (DN3) stage of T-cell development (lckCre Dicer ${ }^{\Delta / \Delta}$ mice) had markedly reduced levels of the miRNAs miR-181, miR-16 and miR-142s in both total thymocyte populations and double positive (DP) thymocytes. Thymic cellularity in the lckCre Dicer ${ }^{\Delta / \Delta}$ mice was also markedly reduced compared with mice expressing functional Dicer.
Interestingly, this decrease was mainly a consequence of a reduction in the number of $\alpha \beta-\mathrm{TCR}^{+}$thymocytes, whereas there were normal (or even slightly increased) numbers of DN thymocytes and $\gamma \delta$-TCR ${ }^{+}$thymocytes. Further analysis of thymocytes isolated from lckCre Dicer ${ }^{\Delta / \Delta}$ mice showed that they were less able to survive in vitro than thymocytes from mice expressing functional Dicer.

Despite the decreased number of DP thymocytes in the lckCre Dicer ${ }^{\Delta / \Delta}$ mice, the frequency of cells being positively selected was normal. Furthermore, those few cells that progressed to the $\mathrm{CD} 4^{+}$or $\mathrm{CD} 8^{+}$ single-positive stage transcribed lineage-specific genes - T-helperinducing POZ/Krüppel-like factor (Th-POK; also known as cKROX and ZFP76) and cathepsin W, respectively - indicating that CD4/CD 8 lineage choice is not
HAEMATOPOIESIS

\section{Another branch in the tree}

The current model for the lineage commitment of haematopoietic stem cells (HSCs) proposes that the first decisive step is the complete separation of myelopoiesis and lymphopoiesis through the generation of common myeloid progenitors (CMPs) and common lymphoid progenitors (CLPs). But a recent paper in Cell indicates that this might be preceded by a branch point separating cells that have erythroid and megakaryocytic potential from otherwise multipotent progenitors.

HSCs are defined by the phenotype lineage (Lin) ${ }^{-S C A} 1^{+} \mathrm{KIT}^{\mathrm{hi}}$ (LSK) and can be subdivided by their expression of CD34 and FLT3 (fms-related tyrosine kinase 3 ) into a developmental progression of long-term HSCs (LT-HSCs; LSKCD34 FLT3-), short-term HSCs (ST-HSCs; LSKCD $\left.34^{+}{ }^{-}{ }^{-} 3^{-}\right)$and a population of LSKCD $34^{+}{ }^{+}{ }^{-}{ }^{+}{ }^{+}$HSCs. Jacobsen and colleagues first confirmed previous results showing that the LSKFLT ${ }^{+}$population has combined myeloid and lymphoid potential, being able to generate $B$ cells, T cells and granulocyte/monocyte (GM) progenitors under the appropriate culture conditions. So, according to the current model, LSKFLT3 $^{+}$cells should also be able to generate megakaryocytic and erythroid lineages, which are classically considered to be derived, together with GM progenitors, from CMPs.

However, a series of in vitro and in vivo experiments showed that LSKFLT3 ${ }^{+}$cells lack significant megakaryocytic and erythroid potential. Whereas $57 \%$ of single ST-HSCs produced megakaryocytes in response to culture with thrombopoietin, only $2 \%$ of LSKFLT3 ${ }^{+}$cells did so under the same conditions. Similarly, $53 \%$ of ST-HSCs generated erythroid progeny under appropriate culture conditions, compared with only $3 \%$ of LSKFLT $3^{+}$cells. Therefore, LSKFLT3 $^{+}$cells lack in vitro megakaryocytic and erythroid potential beyond that which could be expected from sorting impurities. In vivo transplant experiments following myeloablation were then used to compare the reconstitution capacity of ST-HSCs and LSKFLT3 ${ }^{+}$cells. Whereas more than $\mathbf{8 0}$ megakaryocyte progenitors were generated per transplanted ST-HSC, transplanted LSKFLT $^{+}$cells did not generate detectable numbers of megakaryocytes.
And ST-HSCs substantially contributed to erythrocyte reconstitution in all mice tested, in contrast to a contribution from LSKFLT $^{+}$cells in only 1 of 13 mice.

Consistent with their GM potential, quantitative reverse-transcriptase PCR showed that both ST-HSCs and LSKFLT3 ${ }^{+}$ cells express high levels of mRNA encoding PU.1 and granulocyte colony-stimulatingfactor receptor. By contrast, LSKFLT3 ${ }^{+}$ cells had no expression of GATA1 (GATAbinding protein 1) or erythropoietin receptor and reduced expression of thrombopoietin receptor compared with ST-HSCs, all of which are known to be required for megakaryocyte and erythrocyte development.

The authors therefore conclude that the first HSC restriction step involves the loss of megakaryocytic and erythroid potential by ST-HSCs, which form LSKFLT3 ${ }^{+}$cells with both GM and CLP potential, and a population of megakaryocyte/erythrocyte progenitors. This new model is consistent with the fact that erythrocyte-like myeloid cells appear earlier in evolution and ontogeny than lymphoid cells.

Kirsty Minton

(2) References and links ORIGINAL RESEARCH PAPER Adolfsson, J. et al. Identification of Flt3+ Iympho-myeloid stem cells lacking erythro-megakaryocytic potential: a revised road map for adult blood lineage commitment. Cell 121, 295-306 (2005) FURTHER READING Godin, I. \& Cumano, A. The hare and the tortoise: an embryonic haematopoietic race. Nature Rev. Immunol. 2, 593-604 (2002) 
regulated by Dicer-generated small RNAs.

These data indicate that functional Dicer is required for the generation and survival of $\alpha \beta-\mathrm{TCR}^{+} \mathrm{T}$ cells, although it is not required for CD4/ CD8 lineage choice or for the specification of lineage-specific gene expression. The mechanisms by which Dicer regulates $\alpha \beta$-TCR ${ }^{+}$T-cell development and survival remain to be determined. However, although Dicer has been shown to mediate heterochromatin silencing through the generation of siRNAs, the authors found no evidence of a defect in either constitutive or facultative heterochromatin silencing in DP thymocytes isolated from the lckCre Dicer ${ }^{\Delta / \Delta}$ mice.

Karen Honey

6) References and links ORIGINAL RESEARCH PAPER Cobb, B. S. et al. $T$ cell lineage choice and differentiation in the absence of RNase III enzyme Dicer. J. Exp. Med. 201, 1367-1373 (2005)

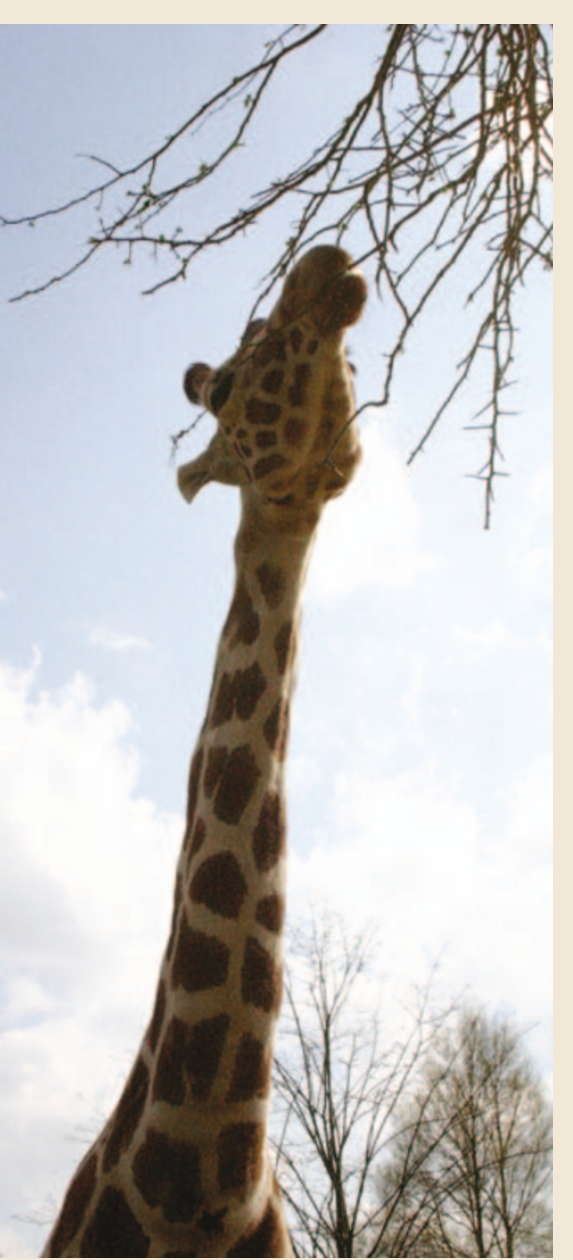

\section{Rolling}

lymphocytes come to an abrupt stop

Before circulating lymphocytes can enter lymph nodes or inflamed peripheral tissues, they must come to a stop on the target endothelium a process known as lymphocyte arrest, which largely depends on activation of the integrin lymphocyte function-associated antigen 1 (LFA1). It is thought that sequential chemokine signals mediate the stepwise activation of LFA1 until a threshold level of active LFA1 is reached. However, this view has been challenged by a report in Nature Immunology indicating that lymphocytes come to an abrupt stop after encountering endothelium-presented chemokines and the LFA1 ligand intercellular adhesion molecule 1 (ICAM1).

LFA1 exists in several conformations: an inactive, bent conformation, which is found at the cell surface of most circulating immune cells; and two extended conformations that have either intermediate or high affinity for ligand, depending on the conformation of the ligand-binding I domain (inserted domain). To assess whether in vivo activation of LFA 1 occurs as a result of an accumulation of successive chemokine-mediated signals, Shamri et al. used intravital microscopy to analyse the behaviour of rolling lymphocytes in high endothelial venules. Surprisingly, rolling lymphocytes were observed to arrest abruptly, rather than to gradually decelerate. Another surprise was that LFA1-dependent arrest occurred in the presence of endothelium-presented chemokines - such as CXC-chemokine ligand 12 (CXCL12) and CC-chemokine ligand 21 (CCL21) - but not after activation by soluble forms of the same chemokines. Similarly, under conditions of shear flow, lymphocytes became arrested in vitro on purified ICAM1 in the presence of immobilized chemokines, but this did not occur after activation by soluble chemokines. Because arrest through LFA1 occurred immediately after encounter with endothelium-presented chemokine, this indicates that LFA1 can be activated within a fraction of a second of contact with co-immobilized chemokine and ICAM1.

Using antibodies specific for distinct LFA1 conformations, it was shown that, under conditions of shear flow, exposure of lymphocytes to the soluble forms of CXCL12 and CCL9 induced the high-affinity conformation of LFA1, which is incompatible with lymphocyte

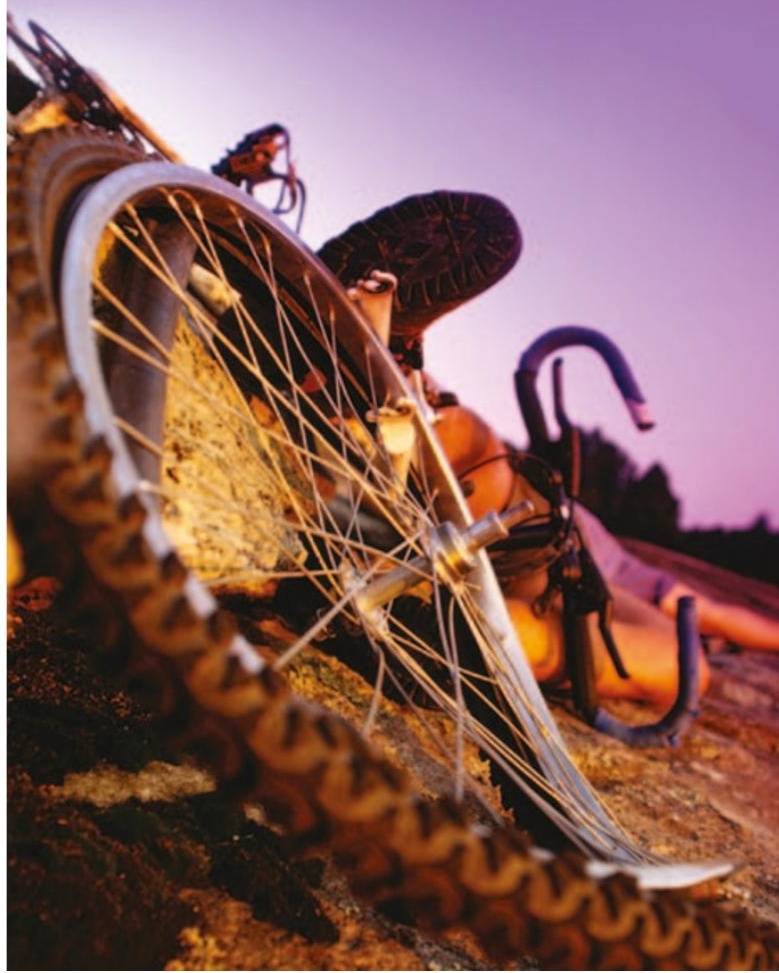

arrest under conditions of shear flow. By contrast, when immobilized, these chemokines induced LFA1 extension to the intermediate conformation but did not generate the highaffinity conformation. Therefore, surprisingly, the ability of chemokines to induce lymphocyte arrest on ICAM1 correlated with their ability to induce extension of LFA1 but not the highaffinity conformation. In further analysis, an inhibitor of the I domain - which prevents the I domain entering the high-affinity conformation (but does not prevent LFA1 entering the extended, intermediate-affinity conformation) - markedly impaired lymphocyte arrest induced by immobilized chemokines. These results indicate that LFA1 extension to the intermediateaffinity conformation can be dissociated from lymphocyte arrest and that the conversion of extended LFA1 to fully activated LFA1 (which is required for lymphocyte arrest) must occur during its very short and localized interaction with ICAM1.

These results indicate that endotheliumimmobilized chemokines induce LFA1 to enter the extended, intermediate-affinity conformation. This primes LFA1 such that it can bind ICAM1, triggering the full activation of the integrin that is required for lymphocyte arrest. This mechanism of lymphocyte arrest is incompatible with the idea that sequential chemokine-mediated signals are required to mediate LFA1 activation in a stepwise manner.

(6) References and links

Karen Honey

ORIGINAL RESEARCH PAPER Shamri, R. et al. Lymphocyte arrest requires instantaneous induction of an extended LFA-1 conformation mediated by endothelium-bound chemokines. Nature Immunol. 6, 497-506 (2005) 